\title{
Title: Narrative literature review on risk factors involved in breast cancer, brain cancer, colon rectal cancer, gynecological malignancy, lung cancer, and prostate cancer.
}

\author{
*Angel Justiz Vaillant, Lyvan Gardiner, Maryam Mohammed, Matthew Surajbally, Luke \\ Maharaj, Luke Ramsingh, Makeisha Simon, Mark Seegobin, Masud Niles \\ *Department of Para-Clinical Sciences. University of the West Indies. Saint Augustine. \\ Trinidad and Tobago. \\ Corresponding author: angel.vaillant@sta.uwi.edu
}

\begin{abstract}
In this study, we describe the most critical risk factors for different malignancies, including breast, prostate, lung, colon-rectal carcinoma, among others and those with risk factors that are modifiable. We revised the literature review about risk factors involved in the genesis of cancer in various databases, including articles indexed in PUBMED, SCOPUS, PMC, and Google Scholar. Knowing risk factors is a mode of fighting malignancy. Diet, lifestyle, practises, and laboratory/clinical interventions were among risk factors of diverse malignancy. We conclude that risk factors can prevent the development of many malignancies in a century where this conundrum is raising disproportionably. It is treated here is to make the public aware of the modifiable risks of cancers.
\end{abstract}

Keywords: Cancer, risk factors, worldwide, lifestyle, practices.

\section{Introduction}

In this study, we examined the risk factors of various types of cancer. It was seen that many activities that are commonly performed in recent times are composed of most of these factors. Whether it is exposure to certain chemicals in the commercial sector, how our foods are processed, or even recreational activities. The result is an upsurge of not only the common types of cancers but also of cancers that were once considered rare, such as melanomas.

The way of life now makes it almost impossible to avoid these issues. Economic constraints and highly competitive job placement for skilled and unskilled labour cause people to endure conditions at job sites that are not good for them. Additionally, from an economic standpoint, processed foods and fast foods/ unhealthy foods are the most important choices. This is due to their affordability or ease of accessibility, and it can be a risk to health.

The peer pressure is another significant factor. Excessive alcohol consumption coupled with smoking can become the norm among the youngest. Finally, it is bad enough to be on an unhealthy work site. However, poor work practices at this job site can worsen the situation. For example, the proper use of personal protective equipment can be safe by isolating them from hazardous chemicals. This would be especially helpful in farming industries, where they are 
foreign, especially for older heads. The main reasons for excessive exposure to these factors can therefore be economically basic or due to a lack of education on the matter. Outstanding malignancy is a disease of a wealthy and old age.

According to the World Health Organization (WHO), cancer incidence will increase by 58\%, from 84,703 cases in 2015 to 133,937 cases in 2035, and cancer mortality will increase by $67 \%$ during this period, from 52,282 to 87,430 deaths (Warner et al., 2018). Nationals of African ancestry exhibited the highest rates of cancer incidence $(243$ per 100,000$)$ and mortality (156 per 100,000) compared to their counterparts in East Indian (incidence: 125 per 100,000; mortality: 66 per 100,000) or mixed ancestry (incidence: 119 per 100,000; mortality: 66 per 100,000) Warner et al.,2018).

\section{Breast cancer}

In 2020, the Centers for Disease Control and Prevention defined breast cancer as an illness in which cells in the breast grow out of control. It can begin in different parts of the breast and metastases, that is lymph nodes vessels. Considering that ravaging affects cancer in one's body, it is essential to identify common risk factors for breast cancer to promote early diagnosis and treatment.

A distinguishing risk factor for breast cancer is the sex of the patient. Breast cancer is more common in women than in women. According to Sun et al. (2017), the number of cases was higher in women $(100 \%)$. The occurrence of breast cancer in men has been described as sporadic, although it is on the rise. Kamińska et al. (2015) explained that breast cancer in men is due to the overexpression of progesterone and estrogen receptors caused by the BCI-2 protein. Both studies also identified age as another critical risk factor, as the risk of breast cancer increases with age. Kamińska et al. (2015) stated that breast cancer is most frequent among postmenopausal women (aged 45 years and older).

In-depth research on genetics of breast cancer done by Sun et al. (2017) and Kamińska et al. (2015) reported that mutations in the BRCA 1 and BRCA 2 genes are linked in an autosomal dominant manner. Sun et al. (2017) discussed the role of other genes, such as overexpression of the HER 2 gene, which has been detected in $20 \%$ of all primary breast cancers. Overexpression of the EGFR gene was also highlighted, along with the fact that it is usually found in cases of inflammatory breast cancer. Additionally, overexpression of the c-Myc gene was discussed, and it was stated that this is typically observed in invasive and non-benign tumors.

Unlike the two studies mentioned earlier, Nazari and Mukherjee (2018) examined the role of mammographic density in breast cancer. According to the study, mammographic density compares the amount of stromal and epithelial tissue with adipose tissue in a woman's breast. Women with a high mammographic density and more stromal and epithelial tissue than adipose tissue have a greater risk of developing breast cancer.

The study identified several factors that influence breast density, one of which is poor dietary choices. Excess fat is an established risk factor due to a diet rich in fat, as stated by Sun et al. 
(2017) and Kamińska et al. (2015), who found that engaging in physical activity at least threefive times per week to minimize fat storage reduces the risk of breast cancer. Kamińska et al. (2015) also discussed the effects of processed foods, stating that products with many chemicals increase the risk, while those containing vitamin D and those with large amounts of antioxidants decrease the risk.

Sun et al. (2017) briefly mentioned other lifestyle choices found to contribute to breast cancer, namely alcohol consumption and smoking. It was reported that $35.4 \mathrm{~g}$ of alcohol daily increases the breast cancer risk (32\%). Nazari and Mukherjee (2018) also found that women whose diets included more than seven servings of alcohol per week had a 17\% higher mammographic density than non-drinkers. However, smoking has been described as a controversial risk because a direct link to breast cancer has not yet been proven.

Sun et al. (2017) failed to mention race as a risk factor, and Kamińska et al. (2015) found that $127.4 \%$ of 100000 Caucasian women develop breast cancer, and $121.4 \%$ in 100000 Black women develop breast cancer. There was a lower incidence among Hispanic patients. However, women of this race are usually diagnosed at a younger age. Nazari and Mukherjee (2018) also referenced studies describing the influence of race and ethnicity on mammographic density. Studies have found the highest density among Asian women and the lowest density among African American women. Furthermore, two studies indicated that breast density was higher in Chinese women than in other ethnic groups.

All three studies identified similar trends in reproductive factors and the risk of breast cancer. Kamińska et al. (2015) found that the first successful pregnancy at an early age, prolonged breastfeeding, and early menopause all lowered the risk. Sun et al. (2017) stated that early menarche, late menopause, and first successful pregnancy at a later age all pose a higher risk.

Estrogen plays a crucial role in breast cancer development. The longer a woman is exposed to estrogen, the higher the risk of cancer, such as early menarche and late menopause. Oral contraceptives and hormone replacement therapy are essential risk factors for breast cancer. Kamińska et al. (2015) and Sun et al. (2017) found it debatable whether oral contraceptives were a risk factor because of conflicting results gained from studies conducted years apart. A possible explanation is that the formulae changed and lowered the risk over time and that women who stopped using oral contraceptives for more than ten years were at a significantly lower risk.

However, Sun et al. (2017) found that the risk was reduced approximately two years after stopping the therapy. Hormone replacement therapy is widely known to increase the mammographic density. However, according to Nazari and Mukherjee (2018), estrogen therapy alone does not cause this, but rather a combination of estrogen and progesterone. Tamoxifen is a drug that blocks estrogen receptors and is associated with decreased mammographic density in the short term, but not in the long term.

In conclusion, age and sex were described as key risk factors for breast cancer, since women aged $>45$ years are at high risk. Specific genes were also found to play an important role, mainly the BRCA 1 and BRCA 2 genes, among others. Women with a higher mammographic density were found to be at a higher risk, as well as those whose diets are rich in fat and those who 
regularly consume alcohol. Studies have found that race and ethnicity are also influential in breast cancer development. Finally, across all studies, it was established that prolonged exposure to estrogen, both endogenous and exogenous, increases the risk of developing breast cancer.

\section{Colon-rectal Cancer}

Colon rectal cancer is a prevalent disease that often causes death. The likelihood of experiencing colorectal malignancy is approximately $4 \%-5 \%$, and the risk of developing CRC is related to individual propensities, such as age, persistent infection history, and way of life. In this review, eight papers were surveyed and analyzed to identify the risk factors associated with colon cancer.

The main risk factor for CRC is age. When a person is over 50 years old, the risk of developing colon cancer is markedly increased. While individuals can develop colon cancer below this age, it is considered rare and marked by familial history or more likely from inherited conditions. of all colon cancers, approximately $2 \%$ to $5 \%$ originate from apparent inherited syndromes, including Lynch syndrome. Despite these conditions, colon cancers show a greater familial risk and are likely to be inherited according to Jasperson et al., 2010.

Furthermore, other inherent risk factors cannot be modified, such as inflammatory bowel disease (IBD) (Stidham \& Higgins, 2018). Patients with IBD showed a significant risk of developing colorectal cancer due to chronic intestinal inflammation's pro-neoplastic effects and family history of CRC (Mármol et al., 2017). Moreover, patients with IBD have a higher risk of developing CRC (Stidham \& Higgins, 2018).

Another risk factor that increased the chances of colon cancer was severe salmonellosis due to Salmonella infection. The results showed an increased risk among patients with salmonellosis for colon cancer, particularly in the ascending and transverse colon. This study concluded that severe salmonellosis resulting from Salmonella infection that can be acquired from contaminated food could increase the risk of developing colon cancer according to Mughini-Gras et al., 2018).

Other risk factors are lifestyle-related and can be altered through diet and physical activity. Diets that are high in red meat consumption have been shown to be carcinogenic according to Mármol et al., 2017. In addition, meat cooked at high temperatures produces heterocyclic amines, which are potential carcinogens. This cohort study acknowledges the relationship between red meat consumption and CRC development. However, we sought to determine the effect of red meat consumption on different parts of the colon and rectum and the effect of the time of consumption on the development of colorectal cancer in both men and women, totaling 134,497 individuals. The results showed that processed meat consumption increases distal CRC risk, while unprocessed red meat consumption has an insignificant effect on the development of proximal cancer. These data have little evidence indicate that increased consumption of unprocessed red meat substantially increases the risk of CRC (Bernstein et al., 2015).

Many studies have focused on alcoholism and the risk of colon rectal carcinoma (Park et al., 2019). The main metabolite of ethanol is acetaldehyde, which is carcinogenic (Mármol et al., 2017). This study confirmed alcohol consumption as a risk factor for colorectal cancer by investigating their association with one another according to ethnicity, sex, lifestyle components, and types of alcoholic drinks. The results showed that those who consumed more than $30 \mathrm{~g}$ of 
alcohol a day increased risk to those who consumed 15-29.9g per day (Park et al., 2019). In addition, the findings stated that beer and wine consumption was directly related to increased colorectal cancer risk, except for liquor.

Tobacco smoking is another lifestyle-related risk that can, in turn, increase the risk of developing colon cancer due to the high content of nicotine, especially in long-term smokers (Centers for Disease Control and Prevention, 2010). This study suggests that vitamin D deficiency is becoming more prevalent globally, with more children and adults at risk of becoming deficient in vitamin D deficiency. This deficiency puts such persons at risk of developing gastrointestinal malignancies. This article also suggests that IBD can also increase the risk of developing colon cancer, as patients with this disease have a higher incidence than the general population. This explains the role of vitamin D in the regulation of immune responses. These functions have been shown to protect against IBD and, thus, inflammation-induced colon cancer (Meeker et al., 2016).

A sedentary lifestyle is thought to contribute to a potential risk factor for colorectal cancer, such as obesity, according to Dong et al., 2017. Different lifestyle habits are considered risk factors for colon cancer, which can be changed through diet and physical activity, while risk factors cannot be modified, such as age and family history of colon cancer (Frezza et al., 2006).

\section{Glyomas (brain cancer).}

In this review, however, we will look specifically at the risk factors of gliomas and, more broadly, cancers of the entire central nervous system. First, we examined the gliomas. Glioma can be defined as a type of tumor in the brain and spinal cord regions within glial cells. Hence its name. Gliomas affect 6 per 100,000 people. However, there is a lack of understanding of the risk factors and nature of the disease. Approximately 5\% of patients are diagnosed as living for more than five years (Ostrom et al., 2014).

Second, we defined the risk factors. We explicitly deal with this topic at hand. A risk factor is any variable linked to the risk. Several risk factors have been considered in the recent years. These include a more prolonged adolescent's age, taller individuals, early obesity, later menarche's age, and more recently, more investigations are being conducted to relate it with technology. Remarkably, the use of cell phones could be considered a risk factor for certain tumors. Due to their nature, they would generally be in close contact with an individual's head.

The first study linked the use of mobile phones to gliomas. It was found that in long-term phone usage, there was a 2.2 chance of developing a low-grade glioma. However, there is little evidence linking it to a more aggressive high-grade glioma. Larger sample sizes and more tests must be conducted to further clarify the phenomenon due to the low radiation level and the more than the ten-year waiting time for results. These studies proved to be quite challenging to conduct (Yang et al., 2017).

The same is true for radiation. Another study was conducted on the effect of non-ionizing radiation (such as that of a phone). It is compared with ionizing radiation (such as the type used in CT scans and radiotherapy). The type of radiation referenced was that of survivors of a 
nuclear attack. In contrast, it was found that ionizing radiation generally increased the probability of developing cancer overall. No conclusions can be drawn regarding gliomas (Ostrom et al., 2014). However, where there was some confirmation was about CT scans of the head. Two case studies were conducted. In comparison, it was found that one CT scan did not affect the development of glioma. Cumulative scans over time. It is worth noting that most of the patient's time progression is tracked by taking more than one scan (Ostrom et al., 2014).

A group of radiologists has determined that the minimum gamma exposure that causes tumor development is approximately $10-50 \mathrm{mSv}$. Between 2 and $15 \mathrm{mSv}$, a CT scan is normally released (Linet et al., 2012); therefore, as you can see, it falls well within range, mostly if multiple scans are performed in a short period as the results are compounding.

Second, the presence of inflammatory factors in the blood has also been shown to increase the risk of glioma and CNS cancers. The results were a combination of 31 studies and 2587 patients. In summary, the studies found increased levels of TNF- $\alpha$, TGF- $\beta$, CRP, IL-6, IL-8, and IL-17 in the blood. The risk of glioma/ CNS cancer is high (Feng et al., 2019).

Additionally, with regards to occupational risk factors. However, no correlation has been found between people in environments with low-frequency magnetic fields (McLaughlin et al., 1987). There was an increase in the risk of gliomas and CNS cancers in people who worked in fields where they may have been exposed to varying amounts of hazardous chemicals. Some examples are farmers, firefighters, people who work in textiles, and other chemical-related industries. It was also found that fishers and people working in the forestry division had a reduced risk of gliomas. This test uses data from 15 million people from five countries (Pukkala et al., 2009). Furthermore, we review studies that support the influence of the environment on brain tumors, such as ionizing radiation, toxins including N-nitroso compounds, pesticides, air pollution, and strikingly brain-ionizing irradiation, are well-established factors, especially during childhood (Vienne-Jumeau et al., 2019).

Next, we studied how handedness (whether the person was right-or left-handed) affected the risk of gliomas, in a large control case study in which there were 1849 glioma cases and 1354 healthy controls. We found that right-handed people had a $65 \%$ higher risk of glioma after appropriate adjustments and calculations were made (Miller et al., 2018).

Lastly, mention was made of Neurofibromatosis (NF). It is a group of genetic disorders that are distinct in nature. People are prone to benign and malignant tumor development in the neurons throughout the body. Children with NF1 may develop low-grade gliomas in the brainstem and optic pathways. Persons with NF2 usually develop low-grade tumors affecting regions such as the spinal cord and cranial nerves (Campian \& Gutmann, 2017).

\section{Gynaecological cancers}

Gynecologic cancers are cancers that arise from the reproductive system (Funston et al., 2018). Gynecological cancers include cervical, ovarian, vulvar, uterine/endometrial, and vaginal cancers. This literature review explores the risk factors for gynecological cancer using articles 
that deal with early prevention and medical intervention. Gynecological cancers are preventable, and the risk can be reduced by making necessary changes to one's life. Early diagnosis and medical interventions are crucial for cancer prevention. These factors were highlighted with some bias in the studies. Common risk factors include smoking and papillomaviruses, which can be sexually transmitted, obesity, and age (Funston et al., 2018). These viruses usually suppress the P53 gene, which prevents tumor growth. Deaths are usually caused by metastases or recurrence.

Middle-aged women had the highest incidence of ovarian cancer. However, there can be a genetic predisposition (Funston et al. Also, 5-15\% of ovarian cancer cases are sporadic. Mutations in BRCA1/BRCA2 are responsible for the development of ovarian cancer, which is the most common genetic predisposition. Some lifestyle factors that can increase ovarian cancer include obesity, long-term hormonal therapy, smoking cigarettes, and any asbestos exposure; even so, approximately $21 \%$ of ovarian cancers are considered preventable (Kuchenbaecker et al, 2017).

Endometrial cancer mostly starts in the inner lining of the endometrium (Funston et al., 2018). Endometrial cancer is a very commonly developed woman, and its prevalence has risen over the past two decades. The risk of endometrial cancer increases with age as early menarche, nulliparity, tamoxifen use and late menopause (Braun et al., 2016). Most cases are sporadic, but $10 \%$ can be genetic. This can be attributed to an increase in estrogen levels without a sufficient increase in progesterone levels in sporadic cases. Endometrial cancer is strongly associated with increased body weight. Thus, the increase in obesity over time can be linked to an increase in endometrial cancer. The most critical sign of endometrial cancer is postmenopausal bleeding, although type II diabetes mellitus may also be a risk factor (Njoku et al., 2020).

Invasive cervical cancer is mostly preventable in developed countries (Funston et al., 2018). However, the death rate remains high internationally. The spread of the disease can be mainly attributed to human papillomavirus (HPV) through genital skin contact. The increased infection rate gives rise to an increased likelihood of developing cervical cancer; this is due to the HPV DNA continuously integrating into the host DNA. Other risk factors include combined hormonal contraception and tobacco smoking. HIV also increases the chance of developing cervical cancer and increases the infection rate of Chlamydia trachomatis and low-risk HPV types can also lead to an increased likelihood of developing cervical cancer. One can vaccinate themselves to HPV to reduce chances, and screening is efficient, as it takes years to develop into an invasive malignant mass.

Vulvar cancer is uncommon (2-5\% of gynecological cancers) and affects postmenopausal women. Vulvar cancer can be asymptomatic; however, the presence of a lump or ulcer can be an indicator. HPV prevalence, HIV positivity, smoking, and inflammation all increase the chances of developing vulvar cancer. Some ways to reduce this risk include vaccination and screening (Funston, et al., 2018).

In the literature, transformation into a mesenchymal phenotype may naturally lead to poor prognosis in gynecological cancers (Lee \& Shen, 2012). Genetic factors in gynecological cancers 
are very important. However, the mechanism and specificity remain in the dark. Through the literature, several studies have shown how the Snail family of transcription factors can play a large role in inducing EMT in ovarian, endometrial, vulvar and cervical cancer (Funston, et al., 2018).

There is information on cardiometabolic risk factors and benign gynecologic disorders. Benign means non-cancerous, but they still play a role in the early diagnosis and prevention of cancers. Cardiometabolic risk can increase the risk of uterine leiomyoma pathogenesis (AlAshqar et al., 2019).

Most of the literature only focuses on the three main types of gynecological cancers: ovarian, uterine/endometrial, and cervical. These three topics have been thoroughly highlighted and have undergone in-depth research, while the less common types of gynecological cancers are left to the wayside. Thus, the increase in cases can be reduced by mitigating risk factors. Genetic factors, such as BRCA1 and BRCA2 germline mutations, are predisposed to cancer (Lynch et al.,1998).

An overview of the literature relating obesity to gynecological factors has been found to not only lack strong links between adiposity and gynecological factors, but that the literature is also biased.

In this research, we see gaps in which only the three main types of gynecological cancers are covered because they have a high mortality rate. However, the steady increase in early diagnosis research, including signs and symptoms, alongside effective screening attempts to mitigate the increasing rates of gynecological cancers, is encouraging.

\section{Stomach cancer}

Gastric carcinoma is the third leading cause of death worldwide. Some risk factors are associated with the development of stomach cancers. It is a literature review on risk factors classified into different categories, such as diet, lifestyle, and infections (Poorolajal et al., 2020).

A diet high in salt intake has generally been linked to a significantly increased risk of stomach cancer. A surplus amount of salt intake serves as a stimulant for the epithelium of the gastric mucosa and causes increased DNA synthesis and cell growth, thus providing the foundation for gastric cancer incidence. Many studies have linked high salt intake with an increased risk of developing stomach cancer. According to these studies, which were reviewed, the OR for salt intake favoured it as a critical risk factor (Poorolajal et al., 2020).

Another dietary risk included the intake of smoked food, which was shown to have a role in gastric carcinogenesis from as far back as the 1960s (Karimi et al., 2014). Inadequate intake of fresh fruits and vegetables is another risk factor for stomach cancer, whereas a higher food intake of fruits and vegetables decreases the risk (González et al., 2006). The next important risk factor for gastric cancer is alcohol consumption. Alcohol consumption increases the intake of nitrosamines by creating a mechanism that causes chronic inflammation. Alcohol consumption has been reported as a risk factor based on 84 studies (Poorolajal et al, 2020). 
Lifestyle habits are another risk factor of gastric carcinoma. A prime example of smoking has been shown to increase the chances of developing gastric cancer in different studies. Smoking has been shown to have devastating and permanent effects on gastric tissue, which increases the malignancy risk of malignancy. Based on the 77 studies reviewed by Poorolajal et al., the overall effect of smoking showed a significantly increased risk of stomach cancer by $61 \%$ (Poorolajal et al., 2020). Family history risks gastric tumours, especially associated first-degree relatives (Yusef et al., 2018), with a higher risk in siblings than in fathers. In addition, these associations are noteworthy for non-cardia cancer rather than cardia cancer (Song et al., 2018).

Different medical conditions and treatments can increase the likelihood of developing stomach cancer. It ranges from the blood group, history of gastrectomy and gastric surgeries, reflex, intestinal metaplasia, and some menstrual and reproductive factors (Yusef et al., 2018). Among the blood groups, $\mathrm{A}+$ was the most common blood group in patients with stomach cancer compared to those with A-. It was found that people with blood type A were $20 \%$ more likely to have gastric cancer than those with blood type O. Nevertheless, further research has shown a significant relationship between type $\mathrm{O}$ blood and gastric cancer before the age of 50 years. In addition to blood groups, a history of gastrectomy and stomach surgery could also increase the chances of developing stomach cancer due to the potential decrease in gastric acid level after surgery and an increase in sensitivity to Helicobacter pylori. Furthermore, chronic atrophic gastritis, history of polyps, gastric ulcer, and family history of esophageal cancers were identified as risk factors for precancerous lesions of gastric lesions, which could lay the foundation for stomach cancer (Yusef et al., 2018).

The next risk factor for stomach cancer is Helicobacter pylori infection. The direct effect of $H$. pylori on stomach epithelial cells induces protein modulation and genetic mutations, leading to inflammation and gastric carcinogenesis. They both work together to promote gastric carcinogenesis. H. pylori mainly causes non-cardia stomach malignancies (Poorolajal et al, 2020).

Gastric cancer is also associated with age and gender factors may play a role in the physiology of gastric carcinoma. Men are more inclined to develop gastric tumours than women. In addition to sex, different occupations may be risk factors. A study on the risk factors of occupational and socioeconomic factors showed that manual workers and farmers had a greater chance of developing gastric cancer. Moreover, cement and mineral dust are considered the most critical occupational risk factors for gastric cancer (Yusef et al., 2018).

Ionizing radiation is another apparent risk factor of gastric cancer. Gamma radiation is also a risk factor. It was concluded that increased sensitivity to gamma rejection was linked to an increased risk of gastric cancer, and persons who were more sensitive to gamma radiation were at a greater risk for stomach cancer (Dong et al., 2012). Stomach cancer is a worldwide concern. Various risk factors can be categorized into different areas. While some modifiable factors can decrease the likelihood of developing stomach, some cannot be modified, such as age and family history. However, the impact of these risks can be diminished through preventative measures and protocols. 


\section{Lung Cancer}

Lung cancer is a common cancer worldwide. Across the globe, it is well known that smoking increases the risk of developing lung carcinoma. However, many other risk factors may contribute to the development of cancer. Some of the less-known risk factors include HIV, bacteria, particulate matter, work-related and environmental hazards, and nutrition.

HIV may cause lung carcinoma due to T-lymphocyte depletion (Sigel et al., 2017). There is evidence that chronic HIV can contribute to the development of lung cancer through HIVspecific mechanisms. HIV is associated with a greater risk of being diagnosed with COPD, which may be due to higher smoking rates in this population and inappropriate immune responses resulting from CD8 overactivity within the lungs, all of which contribute to more significant amounts of inflammation. Recurrent infections can be a compound. All these factors contribute to an increased risk of COPD (Sigel et al., 2017). In addition to dealing with HIV, these patients are further burdened with systemic disparities in their treatment. HIV-positive patients tend to have worse outcomes after being diagnosed with lung cancer. Moreover, they mentioned that a study was conducted to analyze patient outcomes post-surgery for lung cancer patients with HIV and those without and found that those with HIV were prone to more surgical complications along with a lower survival rate post-surgery (Sigel et al., 2017). HIV-infected individuals would benefit significantly from early detection screening and dropping habits commonly associated with lung cancer, such as smoking, as these patient's therapies are not yet optimal. This emphasizes that preventive medicine may have a remarkable effect on lowering patient mortality.

Sigel and colleagues document in their findings that HIV patients are at greater risk when considering some therapeutic options such as adjuvants. They reference a French study that found that chemotherapy treatment for HIV patients resulted in a notable portion of the patients dying, roughly 12\%, figures not seen in their HIV-negative counterparts (Sigel et al., 2017). Among the demographic factors, high smoking rates remain an issue, as smoking has long been established as a significant contributor to the development of lung carcinomas. Interestingly, when examining studies conducted on HIV-positive patients with lung cancer, almost $100 \%$ of them have been reported as smokers. According to these authors in a Danish study, HIV-infected subjects lost a median of 12 years of life due to smoking, while the median years lost was 5.1, for nonsmoker's HIV individuals. The implementation of early detection screening and cessation of smoking will have a profound effect on this demographic's health. It has shown a link between HIV and lung cancer risk due to the high amount of immunosuppression and inflammation present in these patients. To make matters worse, HIV patients are often neglected when it comes to both research and healthcare, resulting in lower survival rates and poorer outcomes.

A correlation between the lung microbiome and cancer has also been established. These bacteria are known as oncobiomes. The lung oncobiome can be described as the sum of microorganisms that reside within the lungs, with the potential to lead to cancer development (Kovaleva et al., 2019). It was found that bacteria can affect immune and tumor cell behaviors. It is seen in the well-known bacterium Mycobacterium tuberculosis, which causes chronic inflammation of lung tissues, which encourages phagocyte activity leading to scar formation.

In addition to Mycobacterium tuberculosis, other bacteria have been found to produce inflammatory responses, although not to the same degree or frequency as TB, these include: Streptococcus pneumoniae, Staphylococcus aureus, Haemophilus influenzae, and Pseudomonas 
aeruginosa, to name a few (Kovaleva et al., 2019). The mechanism by which these organisms cause harm by promoting inflammation increases the activity of the immune system. Heightened immune cell activity changes the composition of the lung biome by altering the population of each bacterial species. In addition to altering the biome itself, increased inflammation results in cell lysis, causing the release of cellular debris.

These compromised cells allow harmful substances to enter the lung tissue, altering the protein concentrations, peptidoglycans, and lipopolysaccharides, leading to harm (Kovaleva et al., 2019). Data has been collected to support their claim that there is a direct relationship between bacteria, immune, and tumor cells to light the mechanisms underlying how these bacteria produce carcinogenic effects. The mechanisms by which bacteria affect cancer progression have been studied for several years, revealing that the significant contributors are bacterial toxins, inflammation, and lung epithelial alteration (Kovaleva et al., 2019). The lung biome is not solely related to cancer development risk, but instead plays an influential role in many physiological processes. It is crucial to consider the consequences of changing the bacterial biome during drug administration. A phenomenon has been described where the effectiveness of checkpoint therapy in cancer patients was drastically lowered in patients with altered microbial populations due to antibiotics (Kovaleva et al., 2019). Studies have proven that administering antibiotics such as vancomycin, ampicillin, and metronidazole can accelerate the formation of neoplasms.

In summary, several microorganisms possess the ability to exacerbate and encourage inflammation. Some of these include Haemophilus influenzae, Escherichia coli, and Pneumococcus (Kovaleva et al., 2019). By promoting inflammatory responses, these organisms can alter the natural environment of the lungs, promote tissue damage, and increase the likelihood of lung cancer development.

Particulate matter is another factor that contributes to lung cancer development. The research looked specifically at the epigenetic changes associated with lung cancer development and environmental factors such as particulate matter on DNA. Epigenetic changes describe alterations that occur at the level of the epigenome that occur in DNA and chromatin to control gene expression and act as an interface between an organism and its environment. Particulate matter is a substance that can be found suspended in air and can vary in size. When considering air pollution as a risk factor for developing lung carcinomas, it is often due to the irritants present in particulate matter. This issue has been brought to the forefront in recent times because of increasing industrialization rates, leading to compromised air quality. According to researchers, the link between pollution and higher lung cancer rates has been found, but the specific role of particulate matter is still under debate ( $\mathrm{Li}$ et al., 2015). The goals of their research were to develop a method for the early detection of lung cancer by examining changes and biomarkers and developing treatment options that would target structures at this level. The epigenetic changes that have been proposed to occur due to particulate matter exposure have been described into three broad categories: modification of histones, methylation of DNA, and changes to micro-RNA. For example, DNA methylation of cytosine in $\mathrm{CpG}$ islands, regions of DNA often resistant to mutations, has been shown to be strongly linked to cancer development (Li et al., 2015).

MicroRNAs, which serve a suppressive regulatory function in gene expression, have been shown to be accompanied by the expression of proteins that are usually repressed. Li et al. stated that miRNAs have various targets that influence the oncogene and tumor suppressor gene levels. 
When looking strictly at lung carcinomas, it has been identified that inadequate let-7 miRNA expression levels have been found to show a strong correlation with the onset of lung cancers. A strong argument was made for the relationship between environmental factors and their direct effect on the epigenome, resulting in epigenetic changes that leave the body more vulnerable to diseases and enabling their progression. These epigenetic changes can be seen in cancer progression and disease state infancy, which allows for the development of possible diagnostic tools (Li et al., 2015).

There is a direct relationship between work-related and environmental exposure to hazardous materials, independent of smoking rates. A study conducted in Korea acknowledges that the population has a high incidence of lung cancer, primarily due to smoking. However, since smoking is a known risk factor, they chose to focus on environmental and occupational factors that contribute to lung cancer development. Emphasis was placed on finding relationships between these occupational and environmental factors within different countries' regions to support their claim. In their report, they stated the existence of work-related causes of cancer, including, but not limited to, aluminum manufacturing, the use of asbestos, industrial metals, exposure to paint, manufacturing with iron and steel, and exposure to high levels of radiation. Low air quality is the primary culprit in the environment and contains carcinogenic particulate matter originating from gas-powered vehicles. Workers in these industries or those exposed to similar conditions are at a greater risk of developing lung cancer (Sung et al., 2018).

By examining the air quality of the various regions over several years, researchers could form conclusive claims that there is an immediate increase in lung cancer risk when exposed to such hazardous environments. Direct relationships between air quality and regions with higher rates of lung cancer were identified. Smoking was intentionally removed as a factor using the standardized rate ratio because it is already a known risk factor (Sung et al., 2018).

The final contributor to the development of lung carcinomas being discussed is nutrition. Researchers aimed to establish a relationship between our diets and the risk of developing lung cancer. They went about this by comparing lung cancer patients to cancer-free individuals of similar backgrounds in Iran. This study was conducted from 2002 to 2005, and the resulting data showed trends for certain foods. These trends were analyzed using conditional logistic regression to determine which foods had protective effects on the participants. The study concluded that most of the tested vegetables and fruits, along with sunflower oil, decreased the risk of lung cancer, while beef, dairy products, bread, vegetable ghee, liver, and animal ghee all showed the potential to increase the risk (Sung et al., 2018).

The risk factors for lung carcinoma are complex and numerous. For a better understanding, further investigation is required, especially in the field of epigenetics. Researchers have discovered that HIV infection leaves patients prone to excessive inflammation, contributing to lung carcinoma development. The natural balance of microorganisms in the lungs plays a vital role in mediating cancer risk. Our environment exposes us to various hazards, such as particulate matter, which can cause epigenetic changes, further increasing our likelihood. Even our dietary choices can prove to be either protective or detrimental.

\section{Prostate cancer (PCA)}

Perdana et al., 2016 assessed the risks associated with prostate carcinoma (PCA). The review stated that the risk factors of PCA include 1 following: 
- Age: $26 \%$ of men over the age of 75 years presented with high-risk disease.

- Ethnicity: Caribbean and South American men have a higher incidence and mortality of PCA than Caucasian men, possibly due to variations in chromosome 8q24 and genes such as BCL2 (an apoptosis gene) and EphB2 (tumor suppression gene).

- Family history - hereditary factors increased prostate epithelial cells' neoplastic transformation with a 2.16-old increased PCA risk when the father or brother was diagnosed with PCA at $<60$ years.

- Elevated insulin-like growth factors have been recognized before prostate cancer diagnosis because of mitogenic and antiapoptotic cell activity.

- STDs - gonorrhea, HPV, or any STD history increases the risk of PCA and causes a three times greater likelihood of death.

- Obesity leads to oncogenesis due to altered levels of metabolic and sex steroid hormones, and the diagnosis of cancer additionally proves to be more difficult because obese men generally have a larger prostate.

- Smoking cigarettes contain carcinogenic chemicals and increase circulating androsterone and testosterone, both of which increase the risk of PCA.

- Alcohol consumption leads to polymorphisms in genes involved in ethanol and folate metabolism and DNA repair.

- A diet high in saturated fat increases insulin growth factors and tumor proliferation, and lipid metabolism generates free radicals, leukotrienes, and prostaglandins, which promote prostate carcinogenesis. Furthermore, red meat, cooking at temperatures between and $125-300{ }^{\circ} \mathrm{C}$, and excessive calcium intake (e.g., dairy products) increases the risk of PCA.

\section{Body mass index and prostate cancer risk.}

A study was conducted with 1,917,430 individuals in Korea and established an association between PCA and obesity based on waist circumference. [2]. The study also explained why there are discrepancies in multiple studies that used body mass index solely to study the association between PCA and obesity.

\section{Race and Ethnicity in prostate cancer.}

A study conducted by Rebbeck TR, 2018, stated that PCA is more common among African Americans than among European Americans. The factors contributing to this outcome are ancestry, genetics and genomics, residential characteristics, and health care. This research compares the PCA's appearance among men of Asian, European, and African descent, stating that PCA is observed in 50\% of Asian men and among younger ages in other populations. Research involving survival disparities among races due to PCA has yielded contradictory findings. Research on the correlation between PCA and genetics has several limitations. Some genes associated with PCA in European, non-European, and African American populations include the HPC1, PCAP, HPCX, CAPB, HPC20, and HOXB13 genes. Those found in African 
American and Japanese populations include 12q24, 1q24-25, 2p16, 2p21, and 1p36. Finally, those explicitly related to African Americans include 2p21, 11q22, 17p11, 22q12, and Xq21 genes [3].

Genome-wide association studies have associated PCA with androgen receptors, kallikrein genes (e.g., KLK3), telomere-related genes (e.g., TERT, TET), matrix metalloprotein genes, microRNA, and loci containing carcinogen metabolism (UGT1A8, CYP21A2). Further studies validated the association of PCA in African Americans with genes such as 8q24, KLK2/3, NUDT10/11, 11q13.2, and HNF1B/TCF2. It was determined that men were at greater risk of PCA if they lived in neighborhoods with a lower socioeconomic status related to education, income, and employment. Studies have shown that African Americans and other ethnicities' care differs in terms of healthcare associated with PCA, resulting in different outcomes of suffering from PCA. Other studies have shown that if equal clinical protocols are provided, the disparity between race groups disappears. Furthermore, African Americans are at greater risk of poorer outcomes since they are less likely to undergo PSA (Prostate-specific antigen) screening than those of European descent [3].

Zhao et al., 2016 concluded a significantly increased relative risk of PCA in individuals who consumed low, medium, high, and higher volumes of alcohol. (Low consumption considered $<24 \mathrm{~g}$ g/day). The study stated that many early research papers did not favor the dose-response relationship, often due to misclassifications, for example, underestimating risks from lowvolume drinking or including former and occasional drinkers, who before the study had experienced declining health, in the abstaining reference group. However, in recent years, more research papers have concluded a link between the volume of alcohol consumed over a long-term period and PCA in recent years. This study attempted to remove bias (previously mentioned) with the inclusion of case-control studies, original articles up till the 14th of December, articles including statistical measurements such as the odds, incidence, and hazard ratios, and utilizing studies with at least three levels of alcohol consumption. Papers with no abstainers and those based on consumption of specific beverages [5].

\section{Incidence and Mortality of Prostate Cancer in Relationship to Family History.}

Abdel-Rahman O, 2019 wrote an article involving 74781 participants, 5381 of whom had firstdegree relatives, and the remaining 69500 participants without a family history (in a first-degree relative). One hundred and $10.5 \%$ of the group without a family history of PCA was subsequently diagnosed with PCA compared to $16.5 \%$ in the group with a PCA family history. Furthermore, the researchers found a correlation between the number of first-degree relatives and PCA incidence, that is. The greater the FDRs, the greater is the PCA incidence of PCA. Family history of PCA was not related to higher PCA mortality in groups that underwent PLCO screening; however, in non-screened groups, a family history of PCA was predictive of higher PCA mortality [6].

\section{Smoking and Prostate Cancer}

De Nunzio et al., 2015 stated that studies have shown a dose-response relationship between smoking and PCA mortality, which is lineal. However, the relationship between cigarette 
smoking and PCA incidence remains unclear. Furthermore, based on observational studies, it has been concluded that smoking leads to worse patient outcomes with PCA treatment with radiotherapy or medical or surgical treatment. When comparing smokers to non-smokers, there was a significant difference in PCA volume and high-grade cancer volume. Additionally, other studies have shown an increased risk of biochemical recurrence and metastasis.

Although the correlation between PCA incidence and cigarette smoking is unclear, biological mechanisms potentially link the two. Mutations in p53 and cytochrome P450 can also occur. Current smokers exhibit higher levels of both total and free testosterone and estradiol levels. There is also inhibition of the aromatase enzyme, leading to less testosterone conversion to estradiol and high testosterone levels. In animal experiments, these increases in testosterone and estrogen levels may promote testosterone-induced carcinogenesis. Furthermore, smoking induces chronic prostate inflammation, which increases the levels of proinflammatory cytokines and inflammatory mediators (De Nunzio et al., 2015).

\section{Influence of Diet and Nutrition on Prostate Cancer}

Matsushita et al., 2020 reported that clinical studies on PCA are controversial. However, it provides insight into how fats, proteins, carbohydrates, vitamins, and polyphenols affect PCA. A study undertaken in a UK population involving 1300 men aged $<60$ years found that a high-fat diet (HFD) was associated with increased PCA incidence compared to a diet with lower lipid intake. An HFD can often result in obesity, which is accompanied by hyperinsulinemia and increased circulating IGF-1 levels. The latter plays a vital role in the development and progression of fat induced PCA. Individuals with a low-fat diet exhibited lower levels of IGF-1, concurrent with PCA growth inhibition and apoptosis in prostate LNCaP cells. High adipocyte quantity leads to elevated CCL2 and CCL2 receptor chemokine receptor 2 in the prostate tissue, causing PCA progression. Finally, epidemiological studies have indicated that the consumption of unsaturated fats instead of saturated fats reduces PCA risk.

The same authors reported that a meta-analytic study comprising 12 articles found that protein intake was not a causative factor of PCA. Other studies have related heterocyclic amines (HCAs) from cooking meat at high temperatures to contribute to PCA development. Clinical findings found that high-fat milk and PCA progression reported a relationship between calcium overdose and PCA risk. Vitamins A, D, and E have been studied in conjunction with the incidence and progression of PCA. Studies have found that vitamin A, a specific lycopene, lowers PCA risk by reducing the gene expression of androgen metabolism and signaling pathways. At moderate levels, vitamin D has a negative association with PCA due to the induced differentiation of immune cells and reduced cancer proliferation. However, high amounts of vitamin D may negatively affect the PCA status (Matsushita et al., 2020).

Other studies have determined that this cancer has a high content of epigallocatechin-3-gallate. EGCG also lowered COX-2 levels in the PCA cells. Furthermore, it downregulates IGF-1 signaling, which has been shown to be associated with PCA incidence. Soy isoflavones, with a structure like 17Beta-estradiol, act as phytoestrogens and bind to the estrogen receptor. Studies 
have shown that they exert antioxidant and inhibitory effects on tyrosine kinase activity, which induces apoptosis in PC3 cells (Matsushita et al., 2020).

Conflict of interest: It does not exist.

Conclusions: Risk Factors can prevent the development of many malignancies in a century where this malignancy is raising disproportionably. What is treated here is to make the public aware of the modifiable risks of cancers.

\section{References}

1. Abdel-Rahman O. (2019). Prostate Cancer Incidence and Mortality in Relationship to Family History of Prostate Cancer; Findings from The PLCO Trial. Clinical genitourinary cancer, 17(4), e837-e844. https://doi.org/10.1016/j.clgc.2019.05.015

2. AlAshqar, A., Patzkowsky, K., Afrin, S., Wild, R., Taylor, H. S., \& Borahay, M. A. (2019). Cardiometabolic Risk Factors and Benign Gynecologic Disorders. Obstetrical \& gynecological survey, 74(11), 661-673. https://doi.org/10.1097/OGX.0000000000000718

3. Bernstein AM, Song M, Zhang X, Pan A, Wang M, Fuchs CS, Le N, Chan AT, Willett WC, Ogino S, Giovannucci EL, Wu K. Processed and Unprocessed Red Meat and Risk of Colorectal Cancer: Analysis by Tumor Location and Modification by Time. PLoS One. 2015 Aug 25;10(8):e0135959. DOI: 10.1371/journal.pone.0135959. PMID: 26305323; PMCID: PMC4549221.

4. Braun, M. M., Overbeek-Wager, E. A., \& Grumbo, R. J. (2016). Diagnosis and Management of Endometrial Cancer. American family physician, 93(6), 468-474.

5. Campian, J., \& Gutmann, D. H. (2017). CNS Tumors in Neurofibromatosis. Journal of clinical oncology : official journal of the American Society of Clinical Oncology, 35(21), 2378-2385. https://doi.org/10.1200/JCO.2016.71.7199

6. Choi, J. B., Myong, J. P., Lee, Y., Kim, I., Kim, J. H., Hong, S. H., \& Ha, U. S. (2020). Does increased body mass index lead to elevated prostate cancer risk? It depends on waist circumference. BMC cancer, 20(1), 589. https://doi.org/10.1186/s12885-020$\underline{07089-5}$

7. Dong Y, Zhou J, Zhu Y, Luo L, He T, Hu H, Liu H, Zhang Y, Luo D, Xu S, Xu L, Liu J, Zhang J, Teng Z. Abdominal obesity and colorectal cancer risk: systematic review and meta-analysis of prospective studies. Biosci Rep. 2017 Dec 12;37(6): BSR20170945. DOI: 10.1042/BSR20170945. PMID: 29026008; PMCID: PMC5725611.

8. Dong, H., Jin, X., Hu, J., Li, H., He, X., Liu, X., \& Bao, G. (2012). High $\gamma$-radiation sensitivity is associated with increased gastric cancer risk in a Chinese Han population: a case-control analysis. PloS one, 7(8), e43625. https://doi.org/10.1371/journal.pone.0043625

9. Feng, Y., Wang, J., Tan, D., Cheng, P., \& Wu, A. (2019). Relationship between circulating inflammatory factors and glioma risk and prognosis: A meta-analysis. Cancer medicine, 8(17), 7454-7468. https://doi.org/10.1002/cam4.2585 
10. Frezza EE, Wachtel MS, Chiriva-Internati M. Influence of obesity on the risk of developing colon cancer. Gut. 2006 Feb;55(2):285-91. doi: 10.1136/gut.2005.073163. Epub 2005 Oct 20. PMID: 16239255; PMCID: PMC1856517.

11. Funston, G., O'Flynn, H., Ryan, N., Hamilton, W., \& Crosbie, E. J. (2018). Recognizing Gynecological Cancer in Primary Care: Risk Factors, Red Flags, and Referrals. Advances in therapy, 35(4), 577-589. https://doi.org/10.1007/s12325-018-0683-3

12. González, C. A., Pera, G., Agudo, A., Bueno-de-Mesquita, H. B., Ceroti, M., Boeing, H., Schulz, M., Del Giudice, G., Plebani, M., Carneiro, F., Berrino, F., Sacerdote, C., Tumino, R., Panico, S., Berglund, G., Simán, H., Hallmans, G., Stenling, R., Martinez, C., Dorronsoro, M., ... Riboli, E. (2006). Fruit and vegetable intake and the risk of stomach and oesophagus adenocarcinoma in the European Prospective Investigation into Cancer and Nutrition (EPIC-EURGAST). International journal of cancer, 118(10), 2559-2566. https://doi.org/10.1002/ijc.21678

13. Hosseini M, Nathan PA, Jafari AM, Yousefifard M, Taslimi S, Khodadad K, Mohammadi F, Sadr M, Rezaei M, Mortaza E, Masjedi MR. Nutrition and lung cancer: a case-control study in Iran. BMC Cancer. 2014 Nov 21;14:860. PMID: 25416035; PMCID: PMC4247167

14. Jasperson KW, Tuohy TM, Neklason DW, Burt RW. Hereditary and familial colon cancers 2010 Jun;138(6):2044-58. doi: 10.1053/j.gastro.2010.01.054. PMID: 20420945; PMCID: PMC3057468.)

15. Kamińska M, Ciszewski T, et al.. Risk factors for breast cancer: Prz Menopauzalny 2015 Sep14(3):196-202. doi: 10.5114/pm.2015.54346. Epub 2015 Sep 30. PMID: 26528110; PMCID: PMC4612558.

16. Kovaleva OV, Romashin D, Zborovskaya IB, Davydov MM et al.: Human lung microbiome on the way to cancer. J Immunol Res. 2019 Jul 29;2019:1394191. DOI: 10.1155/2019/1394191. PMID: 31485458; PMCID: PMC6710786.

17. Kuchenbaecker, K. B., McGuffog, L., Barrowdale, D., Lee, A., Soucy, P., Dennis, J., Domchek, S. M., Robson, M., Spurdle, A. B., Ramus, S. J., Mavaddat, N., Terry, M. B., Neuhausen, S. L., Schmutzler, R. K., Simard, J., Pharoah, P., Offit, K., Couch, F. J., Chenevix-Trench, G., Easton, D. F., ... Antoniou, A. C. (2017). Evaluation of Polygenic Risk Scores for Breast and Ovarian Cancer Risk Prediction in BRCA1 and BRCA2 Mutation Carriers. Journal of the National Cancer Institute, 109(7), djw302. https://doi.org/10.1093/jnci/djw302

18. Lee, M. Y., \& Shen, M. R. (2012). Epithelial-mesenchymal transition in cervical carcinoma. American journal of translational research, 4(1), 1-13.

19. Leitzmann, M. F., \& Rohrmann, S. (2012). Risk factors for the onset of prostatic cancer: age, location, and behavioral correlates. Clinical epidemiology, 4, 1-11. https://doi.org/10.2147/CLEP.S16747

20. Li J, Li WX, Bai C, Song Y. Particulate matter-induced epigenetic changes and lung cancer. Clin Respir J. 2017 Sep;11(5):539-546. DOI: 10.1111/crj.12389. Epub 2015 Oct 20. PMID: 26403658; PMCID: PMC7310573.

21. Linet, M. S., Slovis, T. L., Miller, D. L., Kleinerman, R., Lee, C., Rajaraman, P., \& Berrington de Gonzalez, A. (2012). Cancer risks associated with external radiation from diagnostic imaging procedures. CA: a cancer journal for clinicians, 62(2), 75-100. https://doi.org/10.3322/caac.21132 
22. Lynch, H. T., Casey, M. J., Shaw, T. G., \& Lynch, J. F. (1998). Hereditary Factors in Gynecologic Cancer. The oncologist, 3(5), 319-338.

23. Mármol I, Sánchez-de-Diego C, Pradilla Dieste A, Cerrada E, Rodriguez Yoldi MJ. Colorectal carcinoma: A general overview and future perspectives on colorectal cancer Int J Mol Sci. 2017 Jan 19;18(1):197. doi: 10.3390/ijms18010197. PMID: 28106826; PMCID: PMC5297828.

24. McLaughlin, J. K., Malker, H. S., Blot, W. J., Malker, B. K., Stone, B. J., Weiner, J. A., Ericsson, J. L., \& Fraumeni, J. F., Jr (1987). Occupational risks for intracranial gliomas in Sweden. Journal of the National Cancer Institute, 78(2), 253-257.

25. Meeker S, Seamons A, Maggio-Price L, Paik J. Protective links between vitamin D, inflammatory bowel disease and colon cancer. World J Gastroenterol. 2016 Jan 21;22(3):933-48. DOI: 10.3748/wjg.v22.i3.933. PMID: 26811638; PMCID: PMC4716046.

26. Miller, B., Peeri, N. C., Nabors, L. B., Creed, J. H., Thompson, Z. J., Rozmeski, C. M., LaRocca, R. V., Chowdhary, S., Olson, J. J., Thompson, R. C., \& Egan, K. M. (2018). Handedness and the risk of glioma. Journal of neuro-oncology, 137(3), 639-644. https://doi.org/10.1007/s11060-018-2759-y

27. Mughini-Gras L, Schaapveld M, Kramers J, Mooij S, Neefjes-Borst EA, Pelt WV, Neefjes J. Increased colon cancer risk after severe Salmonella infection. PLoS One. 2018 Jan 17;13(1):e0189721. doi: 10.1371/journal.pone.0189721. PMID: 29342165; PMCID: PMC5771566.

28. Nazari SS, Mukherjee P. Overview of mammographic density and its association with breast cancer breast cancer. 2018 May;25(3):259-267. DOI: 10.1007/s12282-018-0857-5. Epub 2018 Apr 12. PMID: 29651637; PMCID: PMC5906528.

29. Njoku, K., Abiola, J., Russell, J., \& Crosbie, E. J. (2020). Endometrial cancer prevention in high-risk women. Best practice \& research. Clinical obstetrics \& gynaecology, 65, 66-78. https://doi.org/10.1016/j.bpobgyn.2019.12.005

30. Ostrom, Q. T., Bauchet, L., Davis, F. G., Deltour, I., Fisher, J. L., Langer, C. E., Pekmezci, M., Schwartzbaum, J. A., Turner, M. C., Walsh, K. M., Wrensch, M. R., \& Barnholtz-Sloan, J. S. (2014). The epidemiology of glioma in adults: a "state of the science" review. Neuro-oncology, 16(7), 896-913. https://doi.org/10.1093/neuonc/nou087

31. Park SY, Wilkens LR, Setiawan VW, Monroe KR, Haiman CA, Le Marchand L. Alcohol intake and colorectal cancer risk in the multiethnic cohort study. Am J Epidemiol. 2019 Jan 1;188(1):67-76. DOI: 10.1093/aje/kwy208. PMID: 30239578; PMCID: PMC6321802.

32. Perdana, N. R., Mochtar, C. A., Umbas, R., \& Hamid, A. R. (2016). The Risk Factors of Prostate Cancer and Its Prevention: A Literature Review. Acta medica Indonesiana, 48(3), 228-238.2.

33. Poorolajal, J., Moradi, L., Mohammadi, Y., Cheraghi, Z., \& Gohari-Ensaf, F. (2020). Risk factors for stomach cancer: a systematic review and meta-analysis. Epidemiology and health, 42, e2020004. https://doi.org/10.4178/epih.e2020004

34. Pukkala, E., Martinsen, J. I., Lynge, E., Gunnarsdottir, H. K., Sparén, P., Tryggvadottir, L., Weiderpass, E., \& Kjaerheim, K. (2009). Occupation and cancer - follow-up of 15 
million people in five Nordic countries. Acta oncologica (Stockholm, Sweden), 48(5), 646-790. https://doi.org/10.1080/02841860902913546

35. Rebbeck T. R. (2018). Prostate Cancer Disparities by Race and Ethnicity: From Nucleotide to Neighborhood. Cold Spring Harbor perspectives in medicine, 8(9), a030387. https://doi.org/10.1101/cshperspect.a030387

36. Sigel K, Makinson A, Thaler J. Lung cancer in persons with HIV. Current opinion in HIV and AIDS 2017 Jan;12(1):31-38. DOI: 10.1097/coh.0000000000000326.

37. Song, M., Camargo, M. C., Weinstein, S. J., Best, A. F., Männistö, S., Albanes, D., \& Rabkin, C. S. (2018). Family history of cancer in first-degree relatives and risk of gastric cancer and its precursors in a Western population. Gastric cancer : official journal of the International Gastric Cancer Association and the Japanese Gastric Cancer Association, 21(5), 729-737. https://doi.org/10.1007/s10120-018-0807-0

38. Stidham RW, Higgins, PDR Colorectal cancer in inflammatory bowel disease Clin Colon Rectal Surg. 2018 May;31(3):168-178. DOI: 10.1055/s-0037-1602237. Epub 2018 Apr 1. PMID: 29720903; PMCID: PMC5929884.

39. Sun YS, Zhao Z, Yang ZN, Xu F, Lu HJ, Zhu ZY, Shi W, Jiang J, Yao PP, Zhu HP. Risk factors and prevention of breast cancer Int J Biol Sci. 2017 Nov 1;13(11):1387-1397. DOI: 10.7150/ijbs.21635. PMID: 29209143; PMCID: PMC5715522.

40. Sung JH, Sim CS, Ock M, Oh I, Jeong KS, Yoo C. Comparison of a 10-Year Cumulative Age-Standardized Incidence Rate of Lung Cancer among Metropolitan Cities in Korea (During the 2000-2009 Period): Review of Occupational and Environmental Hazards Associated with Lung Cancer. Int J Environ Res Public Health. 2018 Jun 13;15(6):1259. DOI: 10.3390/ijerph15061259. PMID: 29899316; PMCID: PMC6024990.

41. Vienne-Jumeau, A., Tafani, C., \& Ricard, D. (2019). Environmental risk factors of primary brain tumors: A review. Revue neurologique, 175(10), 664-678. https://doi.org/10.1016/j.neurol.2019.08.004

42. Vinceti, M., Malagoli, C., Fiorentini, C., Longo, C., Crespi, C. M., Albertini, G., Ricci, C., Lanzoni, A., Reggiani, M., Virgili, A., Osti, F., Lombardi, M., Santini, M., Fanti, P. A., Dika, E., Sieri, S., Krogh, V., Seidenari, S., \& Pellacani, G. (2011). Inverse association between dietary vitamin $\mathrm{D}$ and risk of cutaneous melanoma in a northern Italy population. Nutrition and cancer, 63(4), 506-513. https://doi.org/10.1080/01635581.2011.539314

43. Warner, W.A., Lee, T.Y., Badal, K. et al. (2018) Cancer incidence and mortality rates and trends in Trinidad and Tobago. BMC Cancer 18, 712. https://doi.org/10.1186/s12885-018-4625-X

44. What Is Breast Cancer? [Internet]. Centers for Disease Control and Prevention. Centers for Disease Control and Prevention 2020 [cited 2020Dec28]. Available from: https://www.cdc.gov/cancer/breast/basic info/what-is-breast-cancer.htm.

45. Yang, K., Fung, T. T., \& Nan, H. (2018). An Epidemiological Review of Diet and Cutaneous Malignant Melanoma. Cancer epidemiology, biomarkers \& prevention : a publication of the American Association for Cancer Research, cosponsored by the American Society of Preventive Oncology, 27(10), 1115-1122. https://doi.org/10.1158/1055-9965.EPI-18-0243 
46. Yang, M., Guo, W., Yang, C., Tang, J., Huang, Q., Feng, S., Jiang, A., Xu, X., \& Jiang, G. (2017). Mobile phone use and glioma risk: A systematic review and metaanalysis. PloS one, 12(5), e0175136. https://doi.org/10.1371/journal.pone.0175136

47. Yusefi, A. R., Bagheri Lankarani, K., Bastani, P., Radinmanesh, M., \& Kavosi, Z. (2018). Risk Factors for Gastric Cancer: A Systematic Review. Asian Pacific journal of cancer prevention : APJCP, 19(3), 591-603.

https://doi.org/10.22034/APJCP.2018.19.3.591

48. Zhao, J., Stockwell, T., Roemer, A., \& Chikritzhs, T. (2016). Is alcohol consumption a risk factor for prostate cancer? A systematic review and meta-analysis. $B M C$ cancer, 16(1), 845. h 16239255; PMCID: PMC1856517. 